\title{
Hacking childhood: Will future technologies undermine, or enable, optimal early childhood development?
}

Hughes $\mathrm{R}^{1}$, Bhopal $\mathrm{S}^{1,2}$, Manu $\mathrm{A}^{3}$, Generative Pre-trained transformer* ${ }^{*}$, Van Heerden $\mathrm{A}^{4,5}$

${ }^{1}$ Maternal \& Child Health Intervention Research Group, Faculty of Epidemiology \& Population Health, London School of Hygiene \& Tropical Medicine, UK

${ }^{2}$ Population Health Sciences Institute, Newcastle University, UK

${ }^{3}$ Ghana Health Service, Ghana

${ }^{4}$ Human Sciences Research Council, Center for Community Based Research, KwaZulu-Natal, South Africa

${ }^{5}$ SAMRC/WITS Developmental Pathways for Health Research Unit Department of Paediatrics, School of Clinical Medicine, Faculty of Health Sciences, University of the Witwatersrand, Johannesburg, South Africa;

It's 2041. Children born during the Covid-19 pandemic are graduating (virtually). For several decades the internet has been part of everyday adult lives. But now, of course, so much more has changed, including for our children. It's almost impossible to tell exactly when we really lost control. Where once, parents (mainly mothers) were considered primary caregivers, now child-rearing is largely the domain of machines guided by the algorithms.

In the home, children are no longer loved and nurtured, but rather treated as commodities to be optimised. A parent's job is not just to love and cherish their child but - working with the machines - to develop them into the best match to the desirable outcomes as possible. While initially controversial, this approach is increasingly evidence-based; a recent study, conducted on a sample of 200,000 babies over 24 months, supported using 12-hour recorded language and emotion-therapy sessions for infants (1). The study showed that the young children were more resilient in later years compared to peers receiving what was known previously as 'play-based childhood development'. Other studies show how these auto-parenting sessions enhance social skills among under 5 year olds (1). Loved ones are now considered an unnecessary threat when it comes to social development - after all they can disrupt even the most carefully structured interactions designed by technical engineers. Some experts argue that it would be preferable to remove parents altogether from daily interactions with young children, because mistakes are all too easy to make(1). After all, parents tend to provide extraneous noise interfering with optimal instruction. 
But the machines taking over was not uncontroversial. Other studies conducted in the 20 s and 30s suggested there may be some things the machines cannot engineer: love, empathy and kindness. Well publicised examples of parents' forgetting their children's names (as they are increasingly anonymously referred to via unique numbers tracked by the National Child Identification Account (NCIA) database(1)) prompted an, albeit short lived, backlash against the rise of automated child rearing. Only time will tell if we have - collectively - made a grave error by out-sourcing child care to the robots.

Back to 2021. An artificial intelligence (Al) engine wrote the previous two paragraphs of this article based on a short prompt ${ }^{1}$. Technology is now much more than "smart" phones and TVs, rather it is about systems that are beginning to mimic human capabilities. Parenting, caregiving, and child development are unlikely to be spared from this revolution. While the wholesale replacement of parents is - thankfully - a long way off, it is worth considering how far and fast we are moving in that direction. That today millions of toddlers and babies interact with Al-powered smart speakers at home (with variable levels of supervision) ought to give us pause for thought.

Complicating the dystopian view articulated above, is the fact that (as with much technological change), existing inequalities are likely to be exacerbated. As the rich access more sophisticated systems, the poor risk being limited to cruder lower-cost versions. Biases are 'baked in' early in development of Al technology, and minimising these requires deliberate action and careful design choices. Given millions of children today lack water, sanitation, toys and human caregivers, will early development be increasingly determined by the digital divide?

But this future has not yet been determined. We feel that those of us with an interest in early childhood can learn, and engage more deeply in the research, development, and regulation of these emerging technologies. We think a less dystopian future is possible. How about this alternative scenario?

Increasing automation means adults work a four day week, spending more time with friends and family. Parents each take two-years paid parental leave. Technology frees us from administration, paying bills and routine shopping. Healthcare appointments including examinations and tests are often conducted from home, and there is more time for families to spend together, playing, reading, singing, laughing and talking.

Whilst there were major concerns regarding privacy of new baby technologies, these were rectified through a series of well thought through privacy conventions of the 2020s.Parents now feel comfortable using privacy-preserving 'federated Al' (2) and microphones, cameras, ingestibles and more to track their baby's movements, speech, cognition and development. Accelerometers, handed out for free in well-baby visits, let parents know when their child hasn't done much rolling or crawling for a few days, and audio/video sensors coach them to develop playful "serve-and-return" (3) interactions which were so easy to forget to do, even when we did have the time, in the old days. And when it comes to learning, early Al voice-recognition 
technologies like SoapBox (www.soapboxlabs.com/educate/) are now evidence-based, inclusive and support children to read, whatever their starting point.

The combination of these 'augmented parenting' technologies and whole-society support for families mean populations are thriving, children are developing and learning more than ever, and even levels of disruptive behaviour and violence are falling in schools as improvements in early childhood development feed through into later childhood. Passive and active sensing devices let parents and health services know about emerging developmental issues like hearing loss, movement problems and language delay earlier than ever. Interventions are delivered by highlytrained, well-paid and motivated professionals supported by helpful technology. Freed by technology from routine work, long professional home visits support exploration of the finer details of child health and development, during which the emphasis is placed on the value of human-tohuman play, love and touch, and how shared attention, love and joy cannot ever be automated.

\section{How can we help to shape the future?}

As academics, we can better engage with these emerging technologies. The changes they will bring are too important to be left to 'big tech', and ought to be shaped rather than followed. The technologies are here, and are evolving fast. Already the LENA (Language Environment Analysis) is showing real-world potential in understanding and tracking vocabulary size, language exposure, and turn-taking - and the public are catching-on (https://www.washingtonpost.com/local/can-a-bit-oftechnology-called-lena-help-young-brains-develop-language/2019/01/01/97d7122e-0ddf-11e9-831f3aa2c2be4cbd story.html

). Left only to companies motivated by profit, the nuances and impacts on inequity are in danger of being sidelined. It is also evident that, like awareness and education about these issues, current regulations for devices and technologies are crude, and out of step with current technologies, let alone where technology will go in the coming decades.

We feel that there is an urgent need for an inclusive and informed conversation about all of these issues. We welcome initiatives like the Lancet-Financial Times Commission on Growing up in a digital world, especially the recognition of "a desperate need to reclaim digital technologies for the good of societies". The creation of a new data-governance model moving away from dataextraction towards trust, solidarity, accountability, and participation feels prescient (5). We feel that the early childhood development community has been insufficiently engaged in these discussions to date. We hope to see more of this in the coming months and years.

It is easy to turn away from these intimidatingly complex debates and discussions; we are all 'out of our depth' in some way here. But they are too important for us to ignore. As they like to say in Silicon Valley, it's time to 'lean in' before early childhood gets 'hacked'.

Footnote: 
${ }^{1}$ These two paragraphs were written by artificial intelligence through providing an autoregressive language model that uses deep learning to generate text (Generative Pre-trained Transformer 3) with the following prompt:

An academic is writing about the future of childhood for publication in the Journal Child Development. He is articulate, thoughtful and clear.

The year is 2040, only half a century on from a time where the internet became part of everyday adult lives, initially through computers and mobile phones. But now, of course, so much more has changed, including for our children.

Co-authors edited this output and incorporated it into the final text. The full tracked-changes version is available at https://bit.ly/3/htKs3 providing an example of the rapidly changing abilities of these technologies.

\section{References:}

1. Imagined and written by co-author Generative pre-trained transformer. Full-text available in appendix: https://bit.ly/3lhtKs3

2. Kaissis GA, Makowski MR, Rückert D, Braren RF. Secure, privacy-preserving and federated machine learning in medical imaging. Nat Mach Intell. 2020 Jun;2(6):305-11

3. National Scientific Council on the Developing Child. The Science of Neglect: The Persistent Absence of Responsive Care Disrupts the Developing Brain [Internet]. Report No.: Working Paper 12. Available from: https://46y5eh11fhgw3ve3ytpwxt9rwpengine.netdna-ssl.com/wp-content/uploads/2012/05/The-Science-of-Neglect-ThePersistent-Absence-of-Responsive-Care-Disrupts-the-Developing-Brain.pdf

4. Code of Federal Regulations. Method For Identifying Toys And Other Articles Intended For Use By Children Under 3 Years Of Age Which Present Choking, Aspiration, Or Ingestion Hazards Because Of Small Parts. Office of the Federal Register; 2011 Jan. Available from: https://www.govinfo.gov/content/pkg/CFR-2011-title16-vol2/pdf/CFR2011-title16-vol2-sec1501-2.pdf

5. Kickbusch I, Agrawal A, Jack A, Lee N, Horton R. Governing health futures 2030: growing up in a digital world-a joint The Lancet and Financial Times Commission. The Lancet. 2019 Oct 12;394(10206):1309. 